\title{
Actividades de Estimación de Medida: La interpretación de los docentes de Educación Primaria*
}

\author{
Measurement estimation activities: The interpretation of Primary School \\ teachers
}

\author{
Noemí Pizarro** \\ ORCID iD 0000-0002-6743-2145 \\ Lluís Albarracín*** \\ ORCID iD 0000-0002-1387-5573 \\ Núria Gorgorió ${ }^{* * * *}$ \\ ORCID iD 0000-0003-3503-9143
}

\begin{abstract}
Resumen
Este artículo presenta la caracterización del conocimiento matemático y didáctico sobre el concepto de estimación de medida que poseen los docentes de primaria chilenos, a partir de la forma en la que proponen utilizar actividades diseñadas y orientadas para trabajar este concepto. Para el análisis utilizamos una definición del concepto de estimación de medida construida a partir del trabajo previo de diversos autores. La metodología utilizada es cualitativa y de carácter descriptivo e interpretativo. Los resultados indican debilidades en el conocimiento de los maestros sobre la estimación de medida y su uso en el aula, constatando que algunos maestros la confunden las actividades de estimación de medida indistintamente con las actividades de medición, o interpretando que son aquellas actividades en las que se puede dar una respuesta aleatoria sin justificación, con lo que se evidencia la necesidad de incidir en la formación del profesorado respecto a la estimación de medida.
\end{abstract}

Palabras clave: Estimación de medida. Conocimiento matemático. Maestros.

\begin{abstract}
* Este trabajo ha recibido el soporte del proyecto EDU2017-82427-R del Ministerio de Economía, Industria y Competitividad (España) y del grupo de investigación LABCOMeC (2017 SGR 00427, Generalitat de Catalunya).

** Doctora en Didáctica de las Matemáticas por la Universitat Autònoma de Barcelona (UAB). Profesora del Departamento de Matemáticas de la Universidad Metropolitana de Ciencias de la Educación (UMCE), Santiago, Región Metropolitana, Chile. Dirección Postal: Doctor Johow 550, Dep, 21B, Ñuñoa, Santiago de Chile, Chile, C.P: 7760210.E-mail: noemi.pizarro@umce.cl.

*** Doctor en Didáctica de las Matemáticas por la Universitat Autònoma de Barcelona (UAB). Professor SerraHúnter en el Dpt. de Didàctica de la Matemàtica i les Ciències Experimentals de la Universitat Autònoma de Barcelona (UAB), Bellaterra, Barcelona, España. Dirección Postal: Campus de la UAB, Edifici G5, 08193, Bellaterra, Barcelona, España. E-mail: 1luis.albarracin@uab.cat.

**** Doctora en Educación por la Universitat Autònoma de Barcelona (UAB). Catedrática en el Dpt. de Didàctica de la Matemàtica i les Ciències Experimentals de la Universitat Autònoma de Barcelona (UAB), Bellaterra, Barcelona, España. Dirección Postal: Campus de la UAB, Edifici G5, 08193, Bellaterra, Barcelona, España. Email: nuria.gorgorio@uab.cat.
\end{abstract}


This article presents the characterization of the mathematical and pedagogical knowledge regarding the measure estimation concept possessed by the Chilean primary school teachers based on the way they propose to utilize activities aimed to work on this concept. In the analysis, we use a definition of the measurement estimation concept constructed from the previous work of different authors. The methodology is of a descriptive and interpretative nature. The results shows weaknesses in the teachers knowledge about the measurement estimate and its use in the classroom, noting that some teachers confuse the measurement estimation activities indistinctly with the measurement activities or interpreting that they are those activities in which a random response can be given without justification, evidencing the need to include measurement estimation in teacher training.

Keywords: Measurement estimation. Mathematical knowledge. Teachers.

\section{Introducción}

En Chile, la última reforma curricular dio a la enseñanza de la medida un nuevo énfasis: ahora es un nuevo eje curricular. De esta forma, el país responde a las recomendaciones de distintos colectivos internacionales (NCTM, 1980; COCKCROFT, 1982; ICMI, 1986) que proponen darle una mayor importancia al tratamiento de las magnitudes.

El eje curricular de Medición tiene como fin que los estudiantes sean capaces de identificar y cuantificar las medidas de los objetos con el fin de poder compararlos y ordenarlos. Se propone iniciar el estudio de la medida con unidades no estandarizadas para, posteriormente, dar paso al estudio de las unidades de medida convencionales de las magnitudes físicas habituales: longitud, área, volumen, tiempo, peso y capacidad (MINEDUC, 2012a). Por otro lado, la medida discreta está presente en el eje de Números, en los dos primeros años de primaria (6 y 7 años), cuando se trabaja el conteo. El plan de estudios de Educación Primaria contempla seis Objetivos de Aprendizaje y doce Indicadores de Evaluación vinculados a la estimación de medida de las diversas magnitudes anteriormente mencionadas.

Considerando lo anterior, se observa que la estimación de medida irrumpe como un nuevo concepto matemático a tratar en las aulas de primaria chilenas. Sin embargo, es altamente probable que el profesorado no tenga preparación para tratarlo. No hay indicios de formación sobre la estimación de medida en la formación escolar, inicial o continua de los docentes. Teniendo en cuenta que los futuros maestros no inician su formación docente con los conocimientos matemáticos adecuados para los contenidos tratados en su formación básica (NORTES; NORTES, 2013), es de esperar que sus conocimientos sobre un contenido no tratado sean deficientes. Sin embargo, el Ministerio de Educación, cada vez que reforma el currículo, publica los Programas de Estudio correspondientes, para que el profesorado comprenda el currículo por medio de actividades, ejemplos y recomendaciones de evaluación. A razón de esta situación curricular, juntamente con las necesidades de conocimiento 
didáctico sobre la estimación de medida, es interesante preguntarse por el conocimiento didáctico y pedagógico de los profesores educación primaria que se enfrentan a las actividades de estimación de medida en el aula proporcionadas por los lineamientos gubernamentales.

\section{Referentes Teóricos}

En esta sección se presentan los conocimientos básicos sobre la estimación como tarea matemática para centrar la atención en la estimación de medida y los conocimientos previos sobre su uso en las aulas.

\subsection{Definiciones del concepto de estimación}

El diccionario de Real Academia Española define el sustantivo estimación como "aprecio y valor que se da y en que se tasa y considera algo" en una definición que se centra en aspectos relacionados con la valoración económica de bienes o servicios (Real Academia Española, 2018, p. 1). Esta definición se aleja del tipo de tareas que asociamos al concepto de estimación en el marco de la Educación Matemática.

En la literatura se pueden observar distintas definiciones sobre estimación, que se corresponden a diferentes tareas relacionadas con distintos ámbitos del saber matemático. Por estimar medida se entiende proceso (BRIGHT, 1976), habilidad (COCKCROFT, 1982; CLAYTON, 1996) o juicio (SEGOVIA et al., 1989), sin embargo, en su caracterización se ha llegado a algunos consensos: estimar consiste en valorar una medida o un cálculo y su respuesta subjetiva (COCKCROFT, 1982; SEGOVIA et al., 1989), involucra trabajo perceptivo (BRIGHT, 1976; CHAMORRO; BELMONTE, 1991) y necesita de la referencia de alguna unidad medida o medida (CLEMENTS; MCMILLEN, 1996; HOGAN; BREZINSKI, 2003; JORAM, 2003; SARAMA; CLEMENTS, 2009).

Es importante señalar que en algunas de las definiciones anteriores no se distingue entre estimación de una medida o un cálculo aritmético, estadístico etc. Desde la psicometría, Hogan y Brezinski (2003) distinguen tres tipos de estimaciones: a) estimación computacional, como proceso por el que se determina rápidamente un valor aproximado para el resultado de una operación aritmética; b) estimación de numerosidades (numerosity), como habilidad de estimar visualmente el número de objetos dispuestos en un plano en un tiempo limitado; y c) estimación de medida, habilidad perceptiva de estimar diferentes magnitudes en objetos 
comunes.

Para Hogan y Brezinski (2003), la estimación computacional es una habilidad que se desarrolla conjuntamente con el resto de habilidades matemáticas en el trabajo relacionado con los contenidos de aritmética. Según estos autores, la estimación de numerosidades y estimación de medida desarrollan y requieren el mismo tipo de habilidades, relacionadas con aspectos perceptivos, y deberían separase desde el punto de vista conceptual de la estimación computacional. De esta forma, podemos observar que estos dos tipos de estimaciones sustentan los objetivos de enseñanza relacionados con la estimación de medida en el marco curricular chileno.

\subsection{Estimación de medida}

Hogan y Brezinski (2003) afirman que el trabajo relacionado con la estimación de medida no está dentro del conjunto de las habilidades matemáticas desarrolladas habitualmente en la escuela y conjeturan, además, sobre la importancia de las imágenes mentales en el desarrollo cognitivo de la medición y la orientación espacial. Clements y McMillen (2009) explican que la apropiación de la cantidad es una referencia de la unidad de medida que debe ser puesta en juego al momento de estimar, dado que una de las características de la estimación es que el individuo posee una herramienta de medición interna, que opera como partición mental o la segmentación de una longitud en una magnitud que no es verbal y que representa una magnitud.

Por otro lado, esta idea de referencia de una medida concreta debe relacionarse con el sentido numérico (JORAM et al., 2005), de este modo, el trabajo con la estimación de medida contribuye al desarrollo de los conceptos de enumeración, número, cantidad y conteo (BRIGHT, 1976; BOULTON-LEWIS; WILS; MUTCH, 1996). En el ámbito sensorial, la estimación de medidas permite desarrollar habilidades perceptivas, puesto que para estimar una medida no se requiere del uso de un instrumento de medición, sino el uso de los sentidos, lo que conlleva reconocer y apoderarse de las unidades de medida (COCKCROFT, 1982; HOGAN; BREZINSKI, 2003). Además, favorece pensamiento de orden superior y las habilidades de resolución de problemas (DOWKER, 1992).

En contraste con los antecedentes anteriores, Chamorro $(1995,1996)$ expresa que los estudiantes rechazan las estimaciones de medida a raíz que, habitualmente, en sus tareas prima la exactitud, incluso en situaciones donde no tiene sentido plantear un problema de precisión. Chamorro (1996) considera que en el escenario educativo se crea una idealización 
de los objetos, donde las mediciones se realizan siempre de forma adecuada y sus resultados son mayoritariamente números enteros, por ello la estimación de medida carece del desarrollo escolar adecuado.

Por su parte, Jones et al. (2012) después de investigar sobre la precisión de las estimaciones de medida de los estudiantes, concluyen que se necesitan más trabajos que incidan en el conocimiento de las habilidades cognitivas relacionadas con la estimación de medida, así como el tipo de actividades necesarias para potenciar su desarrollo en los alumnos. Finalmente, debemos destacar que las habilidades relacionadas con la estimación de medidas han demostrado ser valiosas en diferentes ámbitos laborales (JONES; TAYLOR, 2010) aunque haya sido probado que son difíciles de adquirir tanto para estudiantes como para los profesionales en ejercicio (JORAM; SUBRAHMANYAM; GELMAN, 1998; SOWDER, 1992).

\subsection{Conocimiento didáctico sobre estimación de medida}

Dado que nuestro trabajo se centra en el conocimiento del professor respecto a la enseñanza del concepto de estimación de medida, nos centramos en el marco teórico del Conocimiento Matemático Para la Enseñanza (MKT, siglas que provienen de Mathematical Knowledge for Teaching) propuesto por Ball, Thames y Phelps (2008). El trabajo de estos autores continúa la línea abierta por Shulman (1986) con el Conocimiento del Contenido Específico (SMK) y el Conocimiento Pedagógico del Contenido (PCK) en el ámbito de las matemáticas.

Ball, Thames y Phelps (2008) se proponen determinar el tipo de conocimientos que deben poseer los profesores para enseñar matemáticas de forma eficaz, y se refieren al conocimiento docente necesario para la enseñanza de la matemática. En este estudio se utiliza el trabajo de Ball, Thames y Phelps (2008) para orientar el contenido de las preguntas realizadas en los instrumentos de recogida de datos. Dentro del MKT se definen dos grandes domínios, el del Conocimiento del Contenido y el del Conocimiento Pedagógico del Contenido.

En el domínio del Conocimiento del Contenido se distinguen tres subdomínios: el Conocimiento Común del Contenido (CCK), que es el conocimiento y la habilidad matemática utilizada en entornos distintos de la enseñanza; el Conocimiento del Horizonte HCK); y el Conocimiento Especializado del Contenido (SCK), que es el conocimiento y la habilidad exclusiva de la enseñanza de la matemática. 
En el domínio del conocimiento pedagógico se distinguen los siguientes subdomínios: el Conocimiento del Contenido y los Estudiantes (KCS), que es el conocimiento que combina el conocimiento sobre los estudiantes y la forma en la que aprenden matemáticas; el Conocimiento del Contenido y la Enseñanza (KCT), que combina el conocimiento sobre la enseñanza y el conocimiento sobre las matemáticas; y el Conocimiento del Contenido y el Currículo (Knowledge of Content and Curriculum, KCC).

Los estudios sobre el conocimiento pedagógico de los profesores sobre estimación de medida son escasos, a continuación los destacamos tratando de conectar sus aportaciones con el marco teórico del MKT.

Joram et al. (2005) han indicado que cuando se solicita a un maestro que proponga actividades de estimación de medida a sus alumnos, las tareas solicitadas por los docentes a los estudiantes son adivinanzas en vez de estimaciones, ya que no proporcionan la información o el contexto adecuados para crear el ambiente de trabajo que requiere una estimación. En este caso, el profesorado muestra conocer las características propias de los procesos de estimación de medida, con lo que se pone en evidencia un CCK inadecuado. De igual forma, Forrester y Piké (1998) observaron que la estimación de medida se trataba en las aulas como una hipótesis predictiva, en forma vaga y superflua, carente de respuestas satisfactorias para resolver situaciones a las que sólo podía dar respuesta un instrumento de medida. Estos autores observan que la estimación se trata por medio "del pensamiento sensato" que conlleva a adivinanzas más que juicios de valor a partir de referentes, dejando poca evidencia de la comprensión del concepto y dando cuenta que matemática es sinónimo de rigor y exactitud (FORRESTER; PIKÉ, 1998, p. 346).

Lang (2001) se refirió a la dificultad que tienen los docentes al tratar la estimación de medida, dado que ellos no están seguros de cómo construir un tratamiento de la estimación de medida para que los estudiantes entreguen respuestas razonables. El trabajo de Lang (2001) muestra que las carencias en el CCK son las que motivan las dificultadas en el KCT.

Por su parte y soportándose en un estudio con futuros docentes en un contexto educativo distinto al chileno, Subramaniam (2014) afirma que éstos tienen un conocimiento propio del uso de referentes para afrontar estimaciones de medida en su ámbito personal, pero que este conocimiento no se traduce directamente en un conocimiento didáctico para poder trabajar la estimación de medida en el aula con garantías de éxito. Este resultado pone de manifiesto que es necesario formar a los profesores para poder tratar adecuadamente las actividades de estimación de medida en las aulas, puesto que el dominio en el KCT no implica un adecuado CCK. De hecho, Subramaniam observa una creencia extendida entre los futuros 
docentes: consideran que la única forma de enseñar a realizar estimaciones de medida es proponer actividades prácticas.

\section{Objetivo y contexto del estudio}

El concepto de estimación de medida ha irrumpido en el currículum chileno y, en consecuencia, también en las aulas de primaria (MINEDUC, 2012a). Se da la circunstancia que la estimación de medida es un concepto que no ha sido investigado en profundidad y para el que se han detectado dificultades en el aprendizaje por parte de los alumnos (JONES et al., 2012). Al mismo tiempo, existen estudios que afirman que el profesorado trata las actividades de estimación de medida de forma superficial y que no se siente seguro al trabajar al respecto con los estudiantes (LANG, 2001). Si a lo anterior agregamos que por tradición escolar los profesores chilenos no han tenido formación al respecto, consideramos relevante centrarnos en la forma en la que los docentes interpretan las actividades de estimación de medida propuestas para trabajar en las aulas con los alumnos en los documentos gubernamentales, que deberían ser los principales referentes para ello.

A partir de estas consideraciones, que son las que suponen la situación de partida del estudio, se plantea el siguiente objetivo de investigación:

- Describir y caracterizar el conocimiento matemático y didáctico sobre el concepto de estimación de medida que poseen los docentes de primaria chilenos a partir de la forma en la que proponen utilizar actividades orientadas a trabajar este concepto.

Esta investigación se plantea, además, ser un aporte al estudio del conocimiento didáctico de los profesores, dado que diversos autores han observado carencias en estudios a nivel internacional sobre los conocimientos matemáticos y didácticos del profesorado sobre estimación de medida (CHAMORRO, 1996; FORRESTER; PIKÉ, 1998; HOGAN; BREZINSKI, 2003; CALLÍS et al., 2006; JORAM et al., 2005). Existen dos posibles motivos para explicar esta circunstancia: por un lado, los estudios realizados sobre estimación de medida no poseen la fiabilidad necesaria para ofrecer resultados consistentes (HOGAN; BREZINSKI, 2003) y por otro, es que hay una escasa presencia de materiales para trabajar la estimación de medida, hecho que incide en una presencia testimonial en la formación del profesorado.

\section{Metodología}




\subsection{Población}

Para este estudio se considera encuestar a profesores de primaria chilenos en activo que impartan docencia de matemáticas. Para ello, contactamos tanto con escuelas de primaria como con universidades que imparten formación continua. En total participaron 112 profesores que realizan clases en la enseñanza primaria, con lo que en su práctica docente diaria deben tratar con sus estudiantes algunos de los objetivos de estimación de medida discreta o continua que contempla el currículum.

\subsection{El instrumento de recogida de datos}

Se elabora un cuestionario de preguntas abiertas sobre actividades de estimación de medida. Dado este estudio centrarse en el conocimiento matemático y didáctico que poseen los docentes de primaria sobre estimación de medida, para poder clasificar los diferentes conocimientos que los docentes deberían conocer para tratarla, este estudio se apoya en el MKT de Ball, Thames y Phelps (2008).

Concretamente, nuestras preguntas se enfocan a conseguir información relacionada con el CCK sobre estimación de medida y KCT necesario para guiar el trabajo en el aula para poder entender com mayor detalle la forma es la que se influyen visto que es uno de los aspectos identificados en la literatura, pero para los que no disponemos de suficiente conocimiento. A continuación, presentamos los enunciados de las cuatro preguntas utilizadas para la recogida de datos de este trabajo.

\section{Pregunta 1 (Figura 1)}

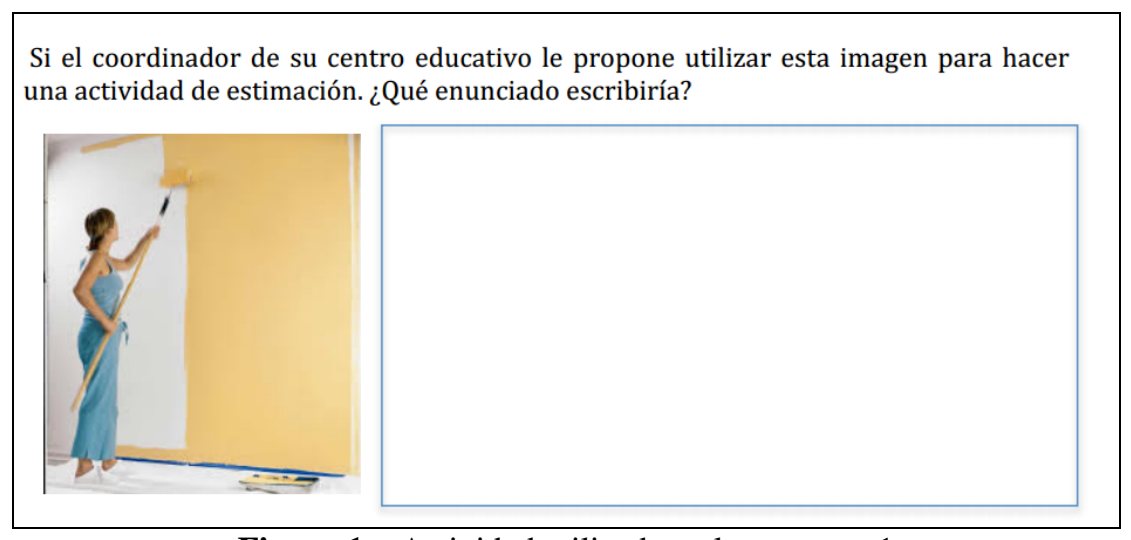

Figura 1 - Actividad utilizada en la pregunta 1 Fuente: Elaboración propia.

La Pregunta 1 tiene como proposito observar qué información consideran necesaria los docentes para trabajar con el concepto de estimación de medida. 
Pregunta 2.a (Figura 2)

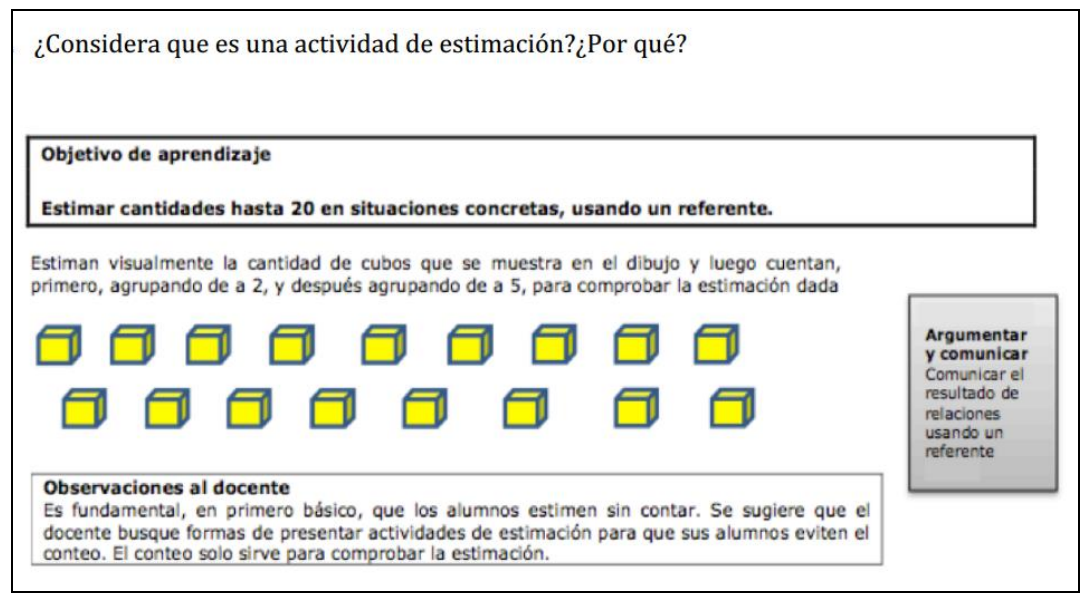

Figura 2 - Actividad utilizada en las preguntas 2.a y 2.b

Fuente: Ministerio de Educación (2012b). Planes y Programas Matemática. Primero Básico. Santiago de Chile:

MINEDUC. http://www.curriculumenlineamineduc.cl/605/w3-propertyvalue-53688.html.

Esta pregunta y las observaciones planteadas son parte de los Planes y Programas para el primer año de primaria (6 años). Pretendemos que los profesores expliquen si consideran que la actividad presentada corresponde o no a una actividad de estimación de medida en la forma en la que está planteada.

Pregunta 2.b: se utiliza la misma actividad que en la pregunta 2.a y se solicita a los docentes que indiquen cómo la utilizarían en el aula, con el fin de observar cómo los docentes evitarían el recuento directo por parte de los estudiantes.

\section{Pregunta 3 (Figura 3)}

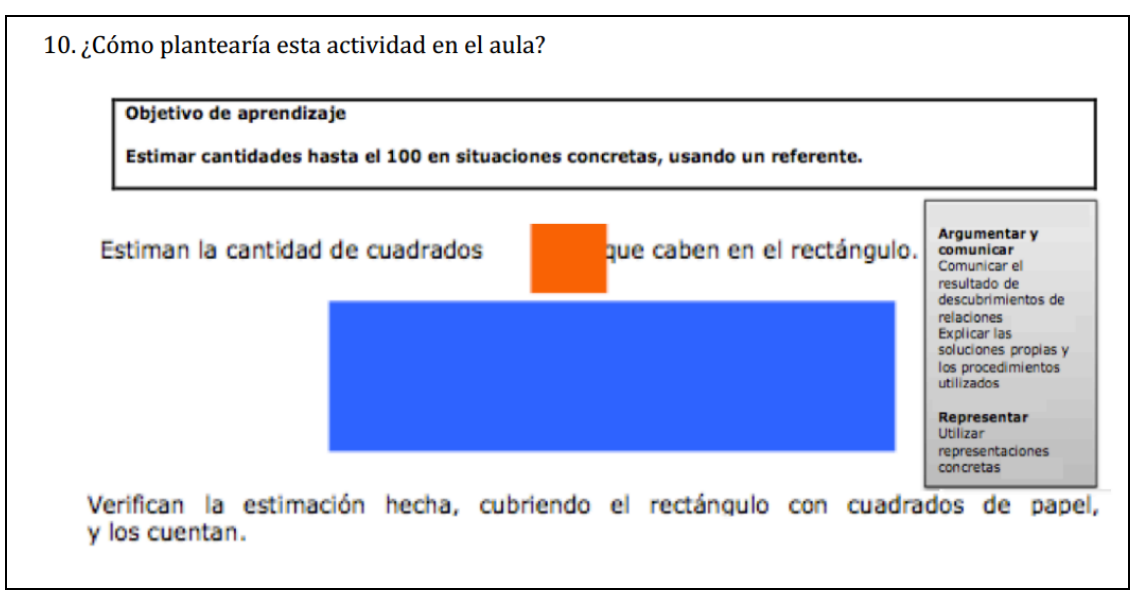

Figura 3 - Actividad utilizada en la pregunta 3

Fuente: Ministerio de Educación (2012b). Planes y Programas Matemática. Primero Básico. Santiago de Chile: MINEDUC. http://www.curriculumenlineamineduc.cl/605/w3-propertyvalue-53688.html.

La pregunta 3 es parte de los Planes y Programas para el segundo año de primaria (7 años). Esta actividad nos permite introducir el uso de un referente explícito como es el cuadrado naranja. 


\subsection{Descripción del proceso de análisis}

En una primera lectura de las respuestas de los docentes constatamos la necesidad de establecer elementos que permitan determinar la naturaleza de las tareas de estimación de medida. Para ello, elaboramos una definición propia del concepto de estimación de medida (PIZARRO; GORGORIÓ; ALBARRACÍN, 2015, 2016), incluyendo en ella los elementos fundamentales que se indican en la literatura revisada.

La definición de estimación de una medida propuesta y utilizada en el proceso de análisis es la siguiente: Asignar perceptivamente un valor o un intervalo de valores y una unidad correspondiente a una cantidad de magnitud discreta o continua por medio de los conocimientos previos o por comparación no directa a algún objeto auxiliar. Esta definición se sustenta tres elementos esenciales:

Asignar un valor numérico (V): para que la tarea pueda considerarse estimación, debe requerir la asignación de un valor numérico. En caso contrario, la tarea sólo es un trabajo perceptivo. Por ejemplo, si al tocar el agua de una piscina afirmamos que es agradable para nadar, la tarea es únicamente una tarea perceptiva, porque no hemos asignado un valor a la temperatura, dado que las unidades de medida no entran en juego. Es indispensable que el estudiante tenga un sentido del ámbito numérico en el que está trabajando (BRIGHT, 1976; COCKCROFT, 1982; SEGOVIA et al., 1989; JORAM et al., 2005; SARAMA; CLEMENTS, 2009).

Realizar la tarea perceptivamente $(P)$ : para estimar es necesario utilizar habilidades perceptivas y evitando el recuento o el uso directo de cualquier instrumento de medida, sea estandarizado o no. En cuanto se usa un instrumento o unidad de medida, haciendo una comparación directa, se está midiendo, no estimando, aunque el resultado de la medición sea aproximado (BRIGHT, 1976; HOGAN; BREZINSKI, 2003; JORAM et al., 2005; SARAMA; CLEMENTS, 2009).

Relacionar la percepción con los conocimientos previos o con la imagen mental de objeto auxiliar $(R)$ : para estimar es necesario tener integrados referentes adecuados o idealmente poseer una noción mental de alguna unidad de medida; en caso contrario, la tarea sería una adivinanza porque no se fundamenta con un valor hecho próprio (CLEMENTS; MCMILLEN, 1996; HOGAN; BREZINSKI, 2003; JORAM, 2003; SARAMA; CLEMENTS, 2009).

Dada la variedad de datos recogidos, esta definición de estimación de medida propuesta no es suficiente para caracterizar la totalidad de las respuestas de los docentes, ya 
que algunas de las respuestas muestran confusiones entre los conceptos de medición y estimación. Ambos procesos tienen como objetivo común dar un valor para una cierta cantidad de medida, pero existen aspectos diferenciadores entre ellos.

En un proceso de medida directo se compara la magnitud a medir con un patrón definido a priori, que actúa como unidad a partir del uso de un instrumento de medida. En cambio, en un proceso de estimación no existe una comparación directa con un instrumento de medida, sino que se utiliza un referente mental adecuado para la magnitud a estimar.

Al estimar se pueden usar dos tipos de referentes: propio o auxiliar. El referente es propio cuando tenemos interiorizada una unidad de medida y es auxiliar cuando tenemos una imagen física de la unidad a utilizar para estimar la medida de la cantidad de magnitud que no puede ser aplicada directamente sobre la magnitud a estimar.

\subsection{Identificación de tareas promovidas}

A continuación se muestra la forma en la que se han analizado los datos para determinar la caracterización de las respuestas de los docentes en relación al conocimiento matemático y didáctico que manifiestan. Las tareas propuestas por los docentes promueven los dos caminos para encontrar el valor de una medida: medición y estimación. Hay docentes que elaboran una pregunta de estimación de medida basada en percepciones y referentes (propios y auxiliares). La siguiente respuesta es una muestra de pregunta que propone un maestro para trabajar con la imagen de la pregunta 1:

Estima la cantidad de $\mathrm{m} 2$ que la mujer debe pintar conociendo que la mujer mide 1,8 $\mathrm{m}$. (Maestro101, 2015).

En esta aportación podemos observar que la pregunta realizada requiere percepción visual (P) ya que se soporta en la información proporcionada por la imagen. En este planteamiento el maestro aporta un referente explícito $(\mathrm{R})$ al dar información sobre la altura de la mujer y se requiere una valoración (V) alumno, que se observa al solicitar la asignación de una cantidad de metros cuadrados. De esta forma, esta respuesta incluye los tres elementos característicos que conforman nuestra definición de estimación de medida.

En la pregunta 3, parte de los docentes considera la valoración de la cantidad de cuadrados que caben en el rectángulo como una actividad de carácter perceptivo, utilizando el cuadrado del enunciado como un referente. Consideramos que estas respuestas plantean la actividad como un trabajo de estimación de medida, según nuestra definición, por ejemplo:

Niños y niñas en la pizarra pueden observar un cuadrado rojo y un rectángulo azul. Sin ponerse de pie, como ustedes quieran, desde su puesto, estimen cuántas veces cabe el 
cuadrado rojo en el rectángulo azul, colocando los cuadrados uno al lado del otro. (Maestro085, 2015)

De esta forma, observamos la intención de conseguir una valoración (V) es sustentada por un acto perceptivo $(\mathrm{P})$, ya que el maestro no permite interacción física entre los estudiantes y las representaciones de las figuras involucradas en la actividad, dado que están en la pizarra y ellos no pueden acceder a ella, los estudiantes están obligados a realizar un ejercicio perceptivo por medio de la visualización.

Observamos que algunos docentes introducen el factor tiempo en la actividad, como muestra la siguiente propuesta de uso de la actividad de la pregunta 2:

Juntaría los cubos y los taparía con un papel y les diría a los niños que rápidamente observen los cubos y estimen cuántos creen que hay.

(Maestro033, 2015).

Consideramos que en aquellos casos en lo que el docente se refiere a trabajar rápidamente está expresando el trabajo en un tiempo determinado, con lo que los estudiantes trabajan de forma similar a las propuestas de numerosity. Algunos docentes explicitan una intención de uso de la actividad que va más allá de presentar la actividad a los estudiantes y darles unas instrucciones adecuadas para potenciar el trabajo de estimación de medida en el aula. La siguiente es una propuesta de un maestro para trabajar la actividad de la pregunta 2 en el aula:

Colocaría una cierta cantidad de bolitas dentro de una bolsa, que ellos supieran cuántas hay y posteriormente mostraría diferentes bolsitas de la misma forma, pero con menos cantidades, que deberían determinar.

(Maestro036, 2015).

En esta respuesta, el docente cambia la actividad, introduciendo materiales que requieren una dinámica de aula diferente del uso habitual de los materiales escritos. Si analizamos esta propuesta a partir de los elementos que hemos considerado, observamos que, en primer lugar, utiliza un referente $(\mathrm{R})$ con la bolsa inicial para tener una noción de medida asociada a la cantidad; posteriormente, pretende utilizar otras bolsas para trabajar la estimación a partir de para el uso de la percepción (P), se solicita la valoración (V) de la cantidad por medio de la estimación.

En otras respuestas de los docentes no hemos detectado conjuntamente los tres elementos que caracterizan la estimación de medida. Una muestra es la siguiente propuesta de uso de la actividad de la pregunta 2:

Utilizaría algún referente, por ejemplo, ver el espacio que utilizan 5 cubos con una hoja cortada y estimar cuántas veces cabe esa hoja en el dibujo, considerando que los cubos siguen un orden.

(Maestro015, 2015). 
Podemos observar en esta respuesta que el docente construye el referente por medio del espacio que utilizan cinco cubos $(\mathrm{R})$ y al pedir cuántas veces incluye la valoración (V). En la respuesta del maestro no encontramos indicios de la forma en la que se determinará el espacio que ocupan esos cinco cubos, con lo que cabe la posibilidad de que esté tratando la actividad como una tarea de medida. Una situación similar la encontramos en la siguiente respuesta a la pregunta 3 :

Primero les mostraría este rectángulo y luego a cada niño le entregaría un papel lustre y les pediría que estimen cuántos cuadrados caben en el rectángulo.

(Maestro033, 2015).

En esta respuesta podemos observar que el foco de atención del maestro se encuentra en la cantidad de cuadrados que caben en el rectángulo (V) pero no ofrece ninguna instrucción ni otro tipo de información que permita determinar la forma en la que piensa guiar a la actividad en el aula. De esta forma, no podemos encontrar aspectos perceptivos (ausencia de P) que serían los que permitirían establecer esta respuesta como un uso de la actividad para trabajar la estimación de medida.

Algunos de los docentes exponen en sus respuestas que pretenden tratar la actividad como una tarea de medición utilizando, para ello, el cuadrado como unidad de medida y haciendo explícita la forma en la que pretenden utilizar estos instrumentos, con lo que podemos afirmar que se corresponde con una actividad de medición. Las respuestas de estas categorías muestran una confusión entre el uso de un referente para realizar una estimación y el uso de un instrumento de medida. La siguiente respuesta a la pregunta 3 es un ejemplo de las clasificadas en esta categoría:

Necesitamos saber cuántos cuadrados necesitamos para cubrir el rectángulo. Construimos el rectángulo y con cuadrados iguales a este, cubrimos el rectángulo.

(Maestro022, 2015).

Podemos observar que el docente indica que la superficie se cubra con el cuadrado, es decir se realice un conteo directo (iteración) con el cuadrado construido sobre el rectángulo, de esta forma el referente se transforma en una unidad de medida no estandarizada. En este caso los alumnos llegarán a una valoración (V) que no estará sustentada en un acto perceptivo (Ausencia de P), sino en una medición.

También hemos detectado propuestas que no ofrecen un nivel de detalle suficiente para poder ser clasificadas y que ponen de manifiesto un amplio desconocimiento de los procesos de estimación de medida. En la pregunta 1 hay docentes que proponen un trabajo de cálculo, ya sea de área o proporciones de área. La siguiente es una muestra de este tipo de actividades propuestas: 
Si Pedro ha tardado 3 horas en pintar la mitad de la pared. ¿Cuánto demorará en pintar la mitad de la pared?

(Maestro013, 2015).

La pregunta elaborada por el docente no necesita de la imagen para poder ser respondida, como tampoco la estimación al no considerar ni la percepción ni el uso de una referencia. Por otro lado, algunos docentes consideraron que los estudiantes debían dar un valor para la cantidad de medida estudiada, pero no ofrecen muestras de potenciar un proceso o razonamiento en base al uso de referentes o percepción, dando espacio a una respuesta aleatoria. Una muestra es esta respuesta para la pregunta 2:

Por ejemplo, preguntar cuántos de estos cubos completan una caja (se muestra la caja). Se puede preguntar su sobrarán cubos o si faltarán.

(Maestro008, 2015).

En esta respuesta podemos observar que el docente espera que sus estudiantes estimen, pero no considera relevante que se trabaje la percepción y el uso de una referencia. Algunos docentes se limitaron sólo a responder con qué material trabajarían la actividad, sin proporcionar aspectos relativos al uso concreto que le darían. Este es un ejemplo de respuesta para la pregunta 3:

Entregando a los estudiantes ambas figuras (1 de cada uno) en cartulina. (Maestro041, 2015).

Observamos que el foco de los docentes está en el material que usarán en la actividad y no en cómo estos materiales serán usados. De esta forma, es imposible comprender cómo el docente desarrollaría la actividad en el aula por falta de información.

\section{Resultados}

A continuación, mostramos la cuantificación del tipo de tarea propuesta por los docentes participantes en el estudio. Debido a la diferente naturaleza de las preguntas formuladas, hemos separado los resultados en dos tablas. La Tabla 1 recoge la cantidad y el porcentaje correspondiente de docentes que diseñaron preguntas que responden a distintos tipos de tareas en la pregunta 1.

Tabla 1 - Tareas sugeridas para usar la imagen de la pregunta 1

\begin{tabular}{lllll}
\cline { 2 - 5 } & $\begin{array}{l}\text { Estimación de } \\
\text { medida }\end{array}$ & Calculo & $\begin{array}{l}\text { No explicita } \\
\text { estimación de medida } \\
\text { o medición }\end{array}$ & $\begin{array}{l}\text { Información sin } \\
\text { pregunta }\end{array}$ \\
\hline Pregunta 1 & $68(60,7 \%)$ & $17(15,2 \%)$ & $14(12,5 \%)$ & $13(11,6 \%)$ \\
\hline
\end{tabular}

La Tabla 1 muestra que cerca de una cuarta parte de los docentes elaboran actividades 
que no contienen pregunta o que esta no se refiere a ninguna cantidad relacionada con el contexto proporcionado. Un $15 \%$ de los maestros elabora una pregunta en la que el alumno debe operar para conseguir un resultado pero no existe un proceso de medida y la actividad se limita a un cálculo aritmético.

Este hecho concuerda con un fenómeno didáctico identificado por Chamorro (2003) denominado aritmetización de la medida, en el que los procesos de medida en las aulas se han substituido por actividades del dominio aritmético. En Mengual, Gorgorió y Albarracín (2017) se cuantifica esta aritmetización de la medida para las actividades propuestas por libros de texto de matemáticas de la etapa de Educación Primaria, identificando que un 66,01\% de las actividades de medida propuestas se presentan aritmetizadamente. Finalmente, un $60,7 \%$ de los docentes participantes en este estudio proporcionan una actividad de estimación de medida para la imagen proporcionada en la pregunta 1.

La Tabla 2 muestra la cantidad y el porcentaje correspondiente de docentes que diseñaron preguntas que responden a distintas tareas en la preguntas $2 . \mathrm{b}$ y 3 .

Tabla 2 - Tareas sugeridas para trabajar los contenidos de las preguntas 2.b y 3

\begin{tabular}{llllll} 
& $\begin{array}{l}\text { Uso de la } \\
\text { actividad } \\
\text { como } \\
\text { estimación de } \\
\text { medida }\end{array}$ & $\begin{array}{l}\text { Uso de dos } \\
\text { componentes } \\
\text { de la } \\
\text { estimación de } \\
\text { medida }\end{array}$ & $\begin{array}{l}\text { Uso de la } \\
\text { actividad } \\
\text { como una } \\
\text { valoración sin } \\
\text { razonamiento }\end{array}$ & $\begin{array}{l}\text { Uso de la } \\
\text { actividad } \\
\text { como } \\
\text { medición }\end{array}$ & $\begin{array}{l}\text { Sólo } \\
\text { menciona } \\
\text { los } \\
\text { materiales } \\
\text { utilizados. }\end{array}$ \\
\hline Pregunta 2.b & $20(17,85 \%)$ & $44(39,2 \%)$ & $11(9,8 \%)$ & $3(2,6 \%)$ & $12(10,71 \%)$ \\
\hline Pregunta 3 & $32(28,5 \%)$ & $0(0,0 \%)$ & $23(32,7 \%)$ & $25(22,3 \%)$ & $4(3,6 \%)$ \\
\hline \multicolumn{5}{c}{ Fuente: Elaboración propia. }
\end{tabular}

Entendemos que al ser las dos actividades presentadas en las preguntas 2 .b y 3 más concretas que la de la pregunta 1 , los resultados obtenidos en estas dos preguntas nos permiten diferenciar con mayor precisión el conocimiento de los maestros. Destaca el hecho de que únicamente un $17,85 \%$ (pregunta 2.a) y un $28.5 \%$ (pregunta 3) plantean actividades que cumplen con todos los requisitos para ser consideradas preguntas de estimación de medida.

El hecho de que la actividad de la pregunta 3 se refiera a una magnitud continua presenta dificultades a los maestros para poder plantear preguntas que usen el cuadrado dado como referente, con lo que acaban proponiendo actividades de medida o de valoración sin promover ningún tipo de razonamiento o reflexión. La pregunta 2.b, al presentar una actividad sobre una medida discreta permite a los maestros usar el referente dado de forma diferente a un conteo, que sería un proceso de medición para este tipo de magnitud.

Muchos docentes, sosteniéndose en su KCT, indicaron que la actividad de la pregunta 2.a no es idónea para trabajar estimación de medida, sino conteo. Una muestra de este tipo de 
argumentos es la siguiente, donde el docente demuestra un conocimiento sobre las diferencias entre estimar y realizar recuentos:

No, porque cuando aún la instrucción sea clara, no considera que lo primero que va a hacer el niño es contar los cubos, perdiendo el foco de estimar.

(Maestro091, 2015).

Otros docentes indican que la actividad no es adecuada para trabajar la estimación de medida, dado que es complicado asegurar que existen referentes previos que permitan trabajar la estimación. Una muestra es la siguiente aportación:

No, porque es complejo estimar el total de cubos sino se tiene un rango de referencia. (Maestro028, 2015).

Entre los docentes que se refirieron a la necesidad de crear referentes previos, ninguno de ellos consideró la posibilidad de utilizar esta pregunta para fomentar la creación de referentes. También hemos detectado docentes que argumentan que la actividad permite trabajar la estimación de medida, ya que se debe realizar utilizando la percepción. Al contrario de los argumentos mostrados anteriormente, estos docentes explican que los objetos pueden ser agrupados y posteriormente a ello se puede realizar la estimación.

Sí, porque los estudiantes visualmente pueden aproximar o estimar la cantidad total de cubos sin la necesidad de contar. También pueden agrupar los cubos (de 5) y aproximar el total para luego comprobar con el conteo.

(Maestro077, 2015).

En esta intervención observamos que el docente no observa las condicionantes de aula que pueden transformar la actividad de estimación en una actividad de recuento. Otros docentes valoran la idea de que los alumnos deben ceñirse a las instrucciones proporcionadas por el enunciado, por lo que no consideran que sea necesaria una monitorización del trabajo de los alumnos, como se muestra en la siguiente respuesta a la pregunta 2.a:

Sí, la encuentro buena (la actividad), porque te da un ámbito, más de 10 o menos 10. Si ellos cuentan no estarían siguiendo la instrucción aquí dice estima, no contar. Ahí dice más o menos de 10.

(Maestro064, 2015).

En la Tabla 3 se observa la cantidad y el porcentaje correspondiente de docentes que reconocieron las debilidades de las preguntas dirigidas a la valorar didácticamente las actividades de estimación de la pregunta 2.a:

Tabla 3 - Carencias de las actividades propuestas en los Planes y Programas según los docentes

\begin{tabular}{llll}
\hline $\begin{array}{l}\text { Reconocimiento de } \\
\text { debilidades de la } \\
\text { pregunta }\end{array}$ & $\begin{array}{l}\text { No considera un } \\
\text { posible conteo }\end{array}$ & No responde & $\begin{array}{l}\text { Sin } \\
\text { clasificación }\end{array}$ \\
\hline $41(36,6 \%)$ & $49(43,8 \%)$ & $17(15,2 \%)$ & $5(4,5 \%)$ \\
\hline
\end{tabular}

Fuente: Elaboración propia 
La tabla 3 muestra que los docentes se dividen en dos grandes grupos, los que comprenden que la actividad de la pregunta 2.a tiene debilidades para se tratada como estimación de medida, dado que favorece el conteo, uno a uno o por agrupación. Por otro lado, el grupo mayoritario no considera que los estudiantes podrían contar los elementos presentados en la actividad.

Finalmente, relacionamos las respuestas de cada uno de los docentes durante todo el cuestionario con el fin de indagar en la consistencia de su conocimiento sobre las actividades de estimación de medida que fueron consideradas en este estudio. A cada respuesta adecuada, entendiéndose por adecuada aquella que posee a lo menos dos componentes de nuestra reconstrucción del concepto de estimación, le asignamos un valor 1 , a las restantes un cero. A partir de la suma de las valoraciones de cada respuesta podremos diferenciar a quienes manifiestan consistencia en las respuestas y, por ende, un conocimiento adecuado para tratar en el aula las actividades de estimación de medida (Tabla 4).

Tabla 4 - Número de respuestas adecuadas en el cuestionario Número de respuestas adecuadas durante el cuestionario

\begin{tabular}{lllll}
\hline 4 & 3 & 2 & 1 & 0 \\
$9(8,0 \%)$ & $32(28,6 \%)$ & $35(31,3 \%)$ & $35(31,25 \%)$ & $10(8,9 \%)$ \\
\hline \multicolumn{5}{c}{ Fuente: Elaboración propia }
\end{tabular}

La Tabla 4 muestra que el porcentaje de docentes que son consistentes en sus respuestas sobre el conocimiento manifestado sobre el concepto de estimación de medidas es bajo. Entendemos que esta situación se debe a que el profesorado participante no ha trabajado, de forma explícita, los contenidos de estimación de medida en su formación previa y se basan en conocimientos procedentes de otros dominios. Este hecho debería hacer reflexionar sobre las actuaciones de las autoridades educativas en el momento en el que un concepto matemático se introduce por primera vez en el currículo escolar (PIZARRO; GORGORIÓ; ALBARRACÍN, 2017). En el contexto de este estudio, destacamos que poco más de un tercio de los docentes encuestados han demostrado tener un conocimiento idóneo en a lo menos tres respuestas de las cuatro realizadas.

\section{Conclusiones}

De los resultados de este estudio se puede concluir que los maestros chilenos utilizan diferentes nociones del concepto de estimación de medida en el momento de trabajar y valorar las actividades vinculadas a él. Se observa que 60,7\% de los 112 docentes participantes en la investigación elaboraron una actividad que involucra valoración, percepción y uso de un 
referente al solicitarles una actividad de estimación de medida en la pregunta 1. Sin embargo, cuando se les pregunta sobre la forma de implementar una actividad de estimación de medida en el contexto de la práctica de aula, las respuestas aumentan en diversidad y, en su mayoría, involucran otros conceptos como la medición o el uso de valoraciones numéricas sin promover el razonamiento matemático. Este hecho indica que una parte importante de los docentes no dispone de elementos de conocimiento para identificar qué tareas son las propias de la estimación de una medida y diferenciarlas de las tareas propias de una medición directa o indirecta. Interpretamos este hecho como una deficiencia en el CCK de los maestros sobre estimación de medida.

Cuando los docentes analizan el uso de las dos actividades de estimación de medida de las preguntas 2.a y 3, que podrían prestarse ambas para ser resueltas mediante el conteo, únicamente un tercio de los docentes observó las debilidades en este sentido que presentan las dos preguntas. En contraposición, un 43,8\% de los profesores no observa que, si se presentan diecisiete cubos ordenados en una figura, es posible que los estudiantes cuenten directamente los cubos y que no realicen ningún proceso propio de la estimación de medida. Esto indica que estos docentes no disponen del conocimiento necesario para pautar la actividad de aula a partir del uso de los materiales didácticos proporcionados por el Ministerio de Educación chileno, lo que puede llevar como consecuencia un uso de las actividades propuestas sin un claro objetivo sobre la enseñanza. A la luz de estos resultados, interpretamos que una parte importante del profesorado encuestado (el 40,15\% que identifica adecuadamente un proceso de estimación de medida en una de las cuatro preguntas) dispone de un conocimiento inadecuado sobre estimación de medida. Este hecho amplía las conclusiones de Forrester y Piké (1998) y Joram et al. (2005), que ya habían identificado que algunos profesores tratan la estimación de medida como una valoración sin uso de referentes.

Nuestro trabajo permite añadir un nuevo elemento a las carencias del profesorado, pues pone en evidencia la confusión entre los procesos de estimación de medida y los de la medida directa, que deberían ser parte fundamental del CCK de los maestros como base para fundamentar el conocimiento pedagógico necesario para atender a las necesidades de los alumnos. Entendemos que es plausible que la confusión del conocimiento del contenido y la enseñanza del concepto de estimación de medida sea por su significado común.

Todos los docentes tienen una concepción predefinida del significado de la palabra estimar que proviene de su vida cotidana porque el concepto no estaba presente en el currículo. Por ello interpretamos que estos docentes no consideran necesario reconstruir ese significado cotidiano como un concepto disciplinario propio de las matemáticas, ya que ese 
significado previo no genera ningún conflicto aparente al interpretar los Objetivos de Enseñanza propuestos por el currículo, pero nos hace plantear la posibilidad de que es posible que en las aulas chilenas el concepto no se esté desarrollando de forma idónea debido a esta confusión.

Esta carencia en el CCK se expande al KCT de los maestros. En concreto, en las preguntas 2.b y 3 únicamente un 57,05\% y un 28,5\% identifican adecuadamente los elementos de estimación de medida necesarios para poder utilizar las actividades propuestas como tales. Este hecho muestra que el resto de maestros no dispone del KCT necesario para poder guiar la actividad en el aula sin que se transforme en una actividad de conteo.

A nivel internacional, los estudios sobre el conocimiento sobre estimación de medida de maestros y profesores son escasos, pero todos ellos apuntan en la dirección de identificar deficiencias de conocimiento de los profesores, con lo que interpretamos que las implicaciones de este estudio son aplicables a otros contextos educativos, en los que ya se ha puesto de relevancia que la estimación de medida no está presente en la formación del profesorado. Los resultados del estudio muestran que es necesario que en los planes de formación de maestros se incluya tanto la definición explícita como el uso de la estimación de medida. Entendemos que debe primarse su ejemplificación y su diferenciación tanto de la medida como de la respuesta aleatoria y en base a respuestas reales de estudiantes, dado que ellas deben basarse en referentes personales.

Finalmente, el presente estudio también realiza aportaciones desde el punto de vista teórico. En primer lugar, la concreción de la definición de estimación de medida propuesta, basada en los conceptos de Valoración, Percepción y Referencia crea un vínculo entre el conocimiento sobre estimación de medida recogido en la literatura y los métodos de análisis. Al mismo tiempo, esta definición clarifica y delimita las tareas de estimación de medida de forma que puede ser utilizada en la formación inicial y continua de maestros y profesores y superando el CCK sobre estimación de medida y estableciendo las bases del SCK necesario para diseñar actividades de aula adecuadas para trabajarla.

\section{Referencias}

BALL, D.; THAMES, M. H.; PHELPS, G. Content Knowledge for Teaching: What Makes It Special? Journal of Teacher Education, New York, NY, v. 59, n. 5, p. 389-407, 2008.

BRIGHT, G.W. Estimation as Part of Learning to Measure. In: National Council of Teachers of Mathematics Yearbook, 38, Reston, VA: NCTM, 1976. p. 87-104.

BOULTON-LEWIS, G.; WILS, L.; MUTCH, S. An analysis of young children's strategies and use of 
devices for length measurement. Journal of Mathematical Behavior, v. 15, p. 329-347, 1996.

CALLÍS, J. et al. Estimación métrica longitudinal en la educación primaria. Factores implícitos en la capacidad estimativa métrica. Uno: Revista De Didáctica De Las Matemáticas, Barcelona, v. 43, p. 91-110, 2006.

CHAMORRO, M.; BELMONTE, J. El problema de la medida. Madrid: Síntesis, 1991.

CHAMORRO, M. Aproximación a la medida de las magnitudes en la enseñanza primaria. Uno: Revista De Didáctica De Las Matemáticas, Barcelona, v. 3, p. 31-53, 1995.

CHAMORRO, M. El currículum de medida en educación primaria y ESO y las capacidades de los escolares. Uno: Revista De Didáctica De Las Matemáticas, Barcelona, v. 10, p. 43-62, 1996.

CHAMORRO, M. El tratamiento escolar de las magnitudes y su medida. In: CHAMORRO, M. C. (Ed.). Didáctica de las matemáticas. Madrid: Pearson, 2003. p. 221-244.

CLAYTON, J. G. A criterion for estimation tasks. International Journal of Mathematical Education in Science and Technology, v. 27, n. 1, p. 87-102, 1996.

CLEMENTS, D. H.; MCMILLEN, S. Rethinking "concrete" manipulatives. Teaching Children Mathematics, Reston, VA, v. 2, p. 270-279, 1996.

COCKCROFT, W. H. Mathematics counts. London: Her Majesty’s Stationery Office, 1982.

DOWKER, A. Computational estimation strategies of professional mathematicians. Journal for Research in Mathematics Education, Reston, VA, v. 23, p. 45-55, 1992.

FORRESTER, M. A.; PIKE, C. D. Learning to estimate in the mathematics classroom: A conversation-analytic approach. Journal for Research in Mathematics Education, Reston, VA, v. 29, p. 334-356, 1998.

HOGAN, T. P.; BREZINSKI, K. L. Quantitative estimation: One, two, or three abilities? Mathematical Thinking and Learning, v. 5, n. 4, p. 259-280, 2003.

ICMI. School Mathematics in the 1990s. Cambridge: Cambridge University Press, 1986.

JONES, M. G. et al. Students' Accuracy of Measurement Estimation: Context, Units, and Logical Thinking. School Science and Mathematics, Birmingham, AL, v. 112, n. 3, p. 171-178, 2012.

JONES, M. G.; TAYLOR, A. Developing a sense of scale: Looking backward. Journal of Research in Science Teaching, Reston, VA, v. 46, n. 4, p. 460-475, 2010.

JORAM, E.; SUBRAHMANYAM, K.; GELMAN, R. Measurement estimation: Learning to map the route from number to quantity and back. Review of Educational Research, Washington, DC, v. 68, p. 413-449, 1998.

JORAM, E. Benchmarks as tools for developing measurement sense. In: CLEMENS, D. H.; BRIGHT, G. (Ed.). Learning and teaching measurement 2003 yearbook. Reston, VA: NCTM. 2003. p. 57-67.

JORAM, E. et al. Children's use of the reference point strategy for measurement estimation. Journal for Research in Mathematics Education, Reston, VA, v. 36, n. 1, p. 4-23, 2005.

LANG, F. K. What is a "good guess" anyway? Teaching Children Mathematics, Reston, VA, v. 7, p. 462-466, 2001. 
MENGUAL, E.; GORGORIÓ, N.; ALBARRACÍN, L. Análisis de las actividades propuestas por un libro de texto: el caso de la medida. REDIMAT - Journal of Research in Mathematics

Education, Barcelona, v. 6, n. 2, p. 136-163, 2017.

MINISTERIO DE EDUCACIÓN. Bases Curriculares de primero a sexto año básico. Santiago de Chile: MINEDUC, 2012a.

MINISTERIO DE EDUCACIÓN. Planes y Programas Matemática. Primero Básico. Santiago de Chile: MINEDUC, 2012b.

NCTM. An Agenda for Action: Recommendations for School Mathematics of the 1980s. Reston, VA: NCTM, 1980.

NORTES, A.; NORTES, R. Formación inicial de maestros: Un estudio en el dominio de las matemáticas. Profesorado, Revista de Currículum y Formación del Profesorado, Granada, v. 17, n. 3, p. 185-200, 2013.

PIZARRO, N.; GORGORIÓ, N.; ALBARRACÍN, L. La definición del concepto estimación de medida de los maestros de primaria. In: FLORES, R. (Ed.). Acta Latinoamericana de Matemática Educativa 28. Barranquilla, Colombia: Comité Latinoamericano de Matemática Educativa, 2015. p. 1202-1209.

PIZARRO, N.; GORGORIÓ, N.; ALBARRACÍN, L. Caracterización de las tareas de estimación y medición de magnitudes. Números, Santa Cruz de Tenerife, v. 91, p. 91-103, 2016.

PIZARRO, N.; GORGORIÓ, N.; ALBARRACÍN, L. Irrupción de un concepto en el currículo: profesores de primaria definen y aplican el concepto de estimación de medida. Dilemas

Contemporáneos: Educación, Política y Valores, México, v. 5, n. 1, p. 1-27, 2017.

REAL ACADEMIA ESPAÑOLA. Diccionario de la Lengua Española. $23^{a}$ edición. 2018. Disponible en: <http://dle.rae.es/?id=GsUOXYe>. Acceso: 23 ago. 2018.

SARAMA, J.; CLEMENTS, D. H. Early childhood mathematics education research: Learning trajectories for young children. New York: Routledge, 2009.

SEGOVIA, I. et al. Estimación en cálculo y medida. Madrid: Síntesis, 1989.

SHULMAN, L. S. Those who understand: knowledge growth in teaching. Educational Researcher, v. 15, n. 2, p. 4-14, 1986.

SOWDER, J. Estimation and number sense. In: Grouws, D. (Ed.). Handbook of research on mathematics teaching and learning. New York: Macmillan Publishing Company, 1992. p. 371-389.

SUBRAMANIAM, K. Prospective secondary mathematics teachers' pedagogical knowledge for teaching the estimation of length measurements. Journal of Mathematics Teacher Education, v. 17, n. 2, p. 177-198, 2014.

Submetido em 29 de Novembro de 2017. Aprovado em 04 de Maio de 2018. 